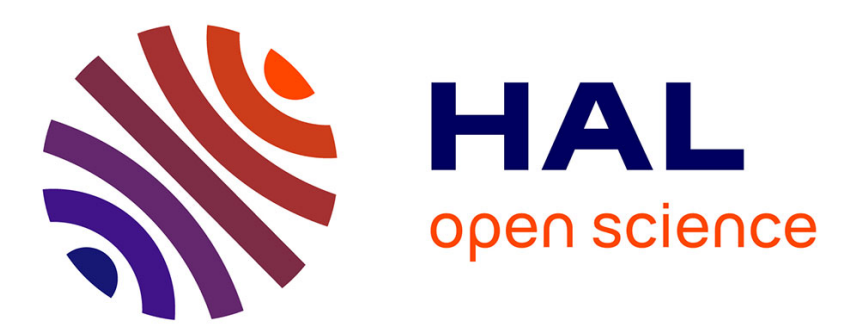

\title{
Jet moléculaire formé par une buse à multicanaux: II. Etude complémentaire
}

\author{
A. Lahmam-Bennani, A. Duguet
}

\section{To cite this version:}

A. Lahmam-Bennani, A. Duguet. Jet moléculaire formé par une buse à multicanaux:

II. Etude complémentaire. Revue de Physique Appliquée, 1979, 14 (3), pp.525-531. 10.1051/rphysap:01979001403052500 . jpa-00244623

HAL Id: jpa-00244623

https://hal.science/jpa-00244623

Submitted on 1 Jan 1979

HAL is a multi-disciplinary open access archive for the deposit and dissemination of scientific research documents, whether they are published or not. The documents may come from teaching and research institutions in France or abroad, or from public or private research centers.
L'archive ouverte pluridisciplinaire HAL, est destinée au dépôt et à la diffusion de documents scientifiques de niveau recherche, publiés ou non, émanant des établissements d'enseignement et de recherche français ou étrangers, des laboratoires publics ou privés. 


\title{
Jet moléculaire formé par une buse à multicanaux : II. Etude complémentaire
}

\author{
A. Lahmam-Bennani et A. Duguet \\ Laboratoire des Collisions Atomiques et Moléculaires $\left({ }^{*}\right)$ \\ Bât. 210, Université de Paris-Sud, 91405 Orsay, France \\ (Reçu le 11 septembre 1978, révisé le 15 novembre 1978, accepté le 20 novembre 1978)
}

\begin{abstract}
Résumé. - L'étude expérimentale antérieure (J. Phys. E 8 (1975) 909) des caractéristiques du jet moléculaire formé par une buse multicanal est reprise et complétée ici par des résultats nouveaux. Dans le domaine de pression d'admission considéré, nous montrons que l'écoulement du gaz ne se fait pas dans un régime moléculaire mais est du type visqueux. Des mesures précises du diamètre angulaire du jet sont présentées, et un accord assez satisfaisant est obtenu avec les calculs de Troitskii. Grâce à une plus grande finesse d'analyse, une conclusion erronée de l'étude précédente est corrigée ici : aucune condensation dans le jet n'est observée jusqu'à des pressions d'admission de 4 bars, permettant ainsi d'obtenir des cibles purement constituées de molécules libres, et dont la densité sur l'axe du jet, à environ $0,5 \mathrm{~mm}$ de la buse, excède $10^{19}$ molécules $/ \mathrm{cm}^{3}$.
\end{abstract}

\begin{abstract}
The previous experimental study (J. Phys. E 8 (1975) 909) of the characteristics of the molecular beam issued from a multichannel nozzle is revisited and completed here by new results. In the investigated pushing pressure range, we show that the gas flow is not molecular but is rather visqueous. Precise measurements of the angular diameter of the gas beam are presented, and satisfactory agreement is obtained when comparing with Troitskii's calculations. Because of different improvements in the analysis technique, an erroneous conclusion of the preceding study is corrected here : no aggregates formation is observed in the gas beam up to 4 bars pushing pressure. One can then obtain a purely molecular target whose density exceeds, on the beam axis, $10^{19}$ molecules/ $\mathrm{cm}^{3}$, at about $0.5 \mathrm{~mm}$ from the nozzle tip.
\end{abstract}

1. Introduction. - Dans une étude expérimentale précédente [1], désignée ci-après par I, nous avions comparé certaines caractéristiques des jets gazeux formés par une buse multicanal et une buse simple. Cependant, divers perfectionnements apportés à notre montage nous ont permis de reprendre cette étude et de la compléter, avec une plus grande précision et une meilleure finesse d'analyse.

2. Type d'écoulement. - Les différents auteurs qui se sont intéressés à l'étude des caractéristiques de la buse multicanal l'ont fait essentiellement, aussi bien du point de vue théorique qu'expérimental, dans le but d'obtenir un jet aussi directif que possible (quelques degrés d'angle d'ouverture), quitte à sacrifier quelque peu l'intensité $[2,3,4]$. Ceci implique nécessairement $[5,6]$ que l'écoulement à la sortie des tubes se fasse en régime moléculaire, c'est-à-dire que la pression $P_{0}$ dans le réservoir en amont de la buse soit

(*) Laboratoire Associé au C.N.R.S. suffisamment faible pour que le nombre de chocs entre molécules devienne négligeable par rapport au nombre de chocs contre les parois. Ceci peut encore se traduire en disant que le libre parcours moyen $\lambda_{0}$ des molécules dans le réservoir doit être :

- soit tel que $\lambda_{0} \gg a$ et $L$, dimensions d'un canal : c'est l'écoulement dit de type Knudsen où les collisions intermoléculaires jouent un rôle négligeable; - soit tel que $a \ll \lambda_{0} \ll L:$ du fait de la chute de pression le long du tube, et donc de l'accroissement de $\lambda$, il existe une région où le libre parcours moyen devient plus grand que ou de l'ordre de grandeur de la longueur de tube restante. Ceci signifie que l'écoulement type Knudsen sera une approximation valable uniquement dans cette longueur restante, dite longueur efficace $L_{\text {eff }}$ : la formation du jet peut alors être considérée comme due à un tube fictif, de longueur $L_{\text {eff }}$, surmonté d'un réservoir fictif caractérisé par un libre parcours moyen $\lambda_{\text {oeff }}$, les collisions intermoléculaires dans ce tube fictif étant négligeables. Dans ce type d'écoulement, la longueur de tube en amont de $L_{\text {eff }}$ joue peu de rôle dans la formation du 
jet et peut plus ou moins être arbitraire. Toutefois il est évident que pour qu'une telle approximation soit valable, il est nécessaire que $L_{\text {eff }} \gg a$.

Dans notre cas, l'intérêt principal de la buse multicanal réside dans l'obtention d'un jet de forte densité, au risque de sacrifier quelque peu la directivité. Pour cela, nous sommes amenés à utiliser des pressions $P_{0}$ relativement fortes dans le réservoir en amont du multicanal ( $P_{0}$ est également appelée pression d'admission), variant usuellement entre 50 torr et 400 torr. Pour les différents gaz que nous avons étudiés $\left(\mathrm{CO}_{2}, \mathrm{CH}_{4}, \mathrm{NH}_{3} \ldots\right)$, le libre parcours moyen $\lambda_{0}$, à $15^{\circ} \mathrm{C}$ et 200 torr, est de l'ordre de grandeur de $16 \times 10^{-5} \mathrm{~mm}$. En comparant aux dimensions des canaux de la buse que nous utilisons $(a=0,1 \mathrm{~mm}$, $L=25 \mathrm{~mm}$ ) on a donc $\lambda_{0} \ll a \ll L$, ce qui signifie que nous ne sommes pas dans les conditions précédemment citées d'un écoulement moléculaire, mais que nous avons affaire à un écoulement visqueux [7] dans lequel les collisions intermoléculaires dans le tube sont prédominantes. Il est bien sûr toujours possible de définir comme plus haut une longueur efficace à partir de laquelle le libre parcours moyen $\lambda$ dans le tube devient équivalent à $L_{\text {eff }}$ : Troitskii [7] a montré que dans ces conditions $L_{\text {eff }}$ pouvait assez bien être représenté par :

$$
L_{\text {eff }}^{3 / 2}=2 \lambda_{0} L^{1 / 2}
$$

ce qui donnerait dans notre cas, pour $\mathrm{CO}_{2}$ à 200 torr, $L_{\text {eff }}=1,37 \times 10^{-2} \mathrm{~mm}$, valeur qui est très petite devant le diamètre a d'un tube. L'écoulement ne peut donc être considéré comme moléculaire, même dans le tube efficace équivalent. Ces considérations semblent impliquer, dans nos conditions expérimentales, un régime visqueux. Une partie de l'étude expérimentale qui suit est consacrée à vérifier cette conclusion.

3. Etude expérimentale. - 3.1 PRINCIPE. - La technique de mesure est identique à celle utilisée en I et ne sera décrite que brièvement : un faisceau d'électrons de $35 \mathrm{keV}$, de diamètre à mi-hauteur $0,275 \mathrm{~mm}$, croise à angle droit le jet de gaz (Fig. 1). Après diffusion, ce faisceau est capté dans un Faraday. Les électrons diffusés sont recueillis par un capteur situé à $400 \mathrm{~mm}$ du centre de collision, et couvrant environ le domaine angulaire $0,5^{\circ}<\theta<7,5^{\circ}$, ce qui correspond à $s_{\min }=0,85 \AA^{-1}$ et $s_{\max }=13 \AA^{-1}$, où $s$ est le paramètre de diffraction $s=\frac{4 \pi}{\lambda} \sin \theta / 2$, et $\lambda$ est la longueur d'onde associée aux électrons à $35 \mathrm{keV}$.

Le rapport de l'intensité diffusée $I$ à l'intensité incidente $I_{0}$ est alors étudié en fonction de la pression d'admission $P_{0}$ et des paramètres $x$ et $y$ définissant la position du faisceau d'électrons par rapport à la buse. En fait, les axes $x$ et $y$ sont mobiles avec la buse, et le faisceau d'électrons est fixe.

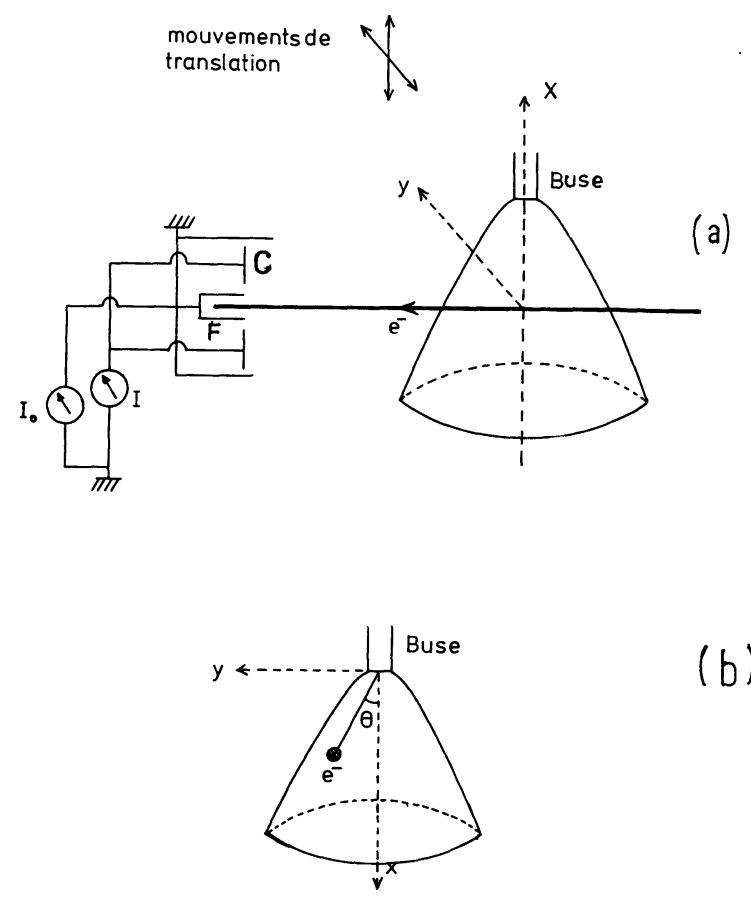

Fig. 1. - a) Schéma de principe de l'étude des caractéristiques du jet issu de la buse. F : Faraday, C : Capteur, utilisés respectivement pour la mesure des intensités électroniques incidente $I_{0}$ et diffusée $I$. $b$ ) Définition du paramètre angulaire $\theta$.

[a) Schematic principle for the study of the characteristics of the gas beam produced by the nozzle. $\mathrm{F}$ is a Faraday and $\mathrm{C}$ is a beam trap, respectively used for the measurement of the incident, $I_{0}$, and the scattered, $I$, electron intensities. $b$ ) Definition of the angular parameter $\theta$.]

3.2 DiAmètre et ANGLE D'OUVERTURE DU JET. En déplaçant la buse suivant l'axe $y$, nous avons déterminé le profil du jet $\left(I / I_{0}\right)_{x, P_{0}}=f(y)$ pour diverses valeurs de $x$, distance de la buse au faisceau d'électrons, et de $P_{0}$, pression d'admission du gaz. Un exemple d'un tel profil est donné sur la figure 2.

Nous avons alors défini le diamètre $\Phi$ du jet comme étant, à $P_{0}$ et $x$ fixés, la largeur à mi-hauteur de tels profils. Les variations de ce diamètre $\Phi$ avec $P_{0}$

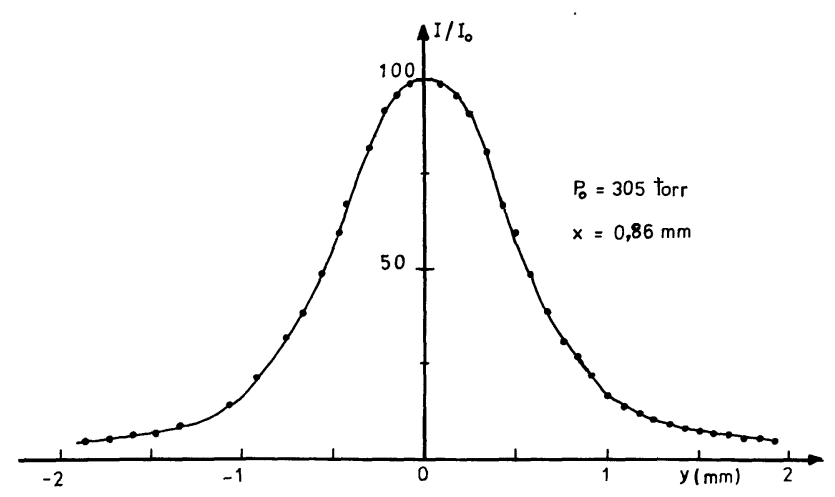

Fig. 2. - Profil du jet de gaz, dans un plan situé à une distance $x$ constante de la buse.

[Gas beam profile in a plane located at a constant distance $x$ from the nozzle.] 


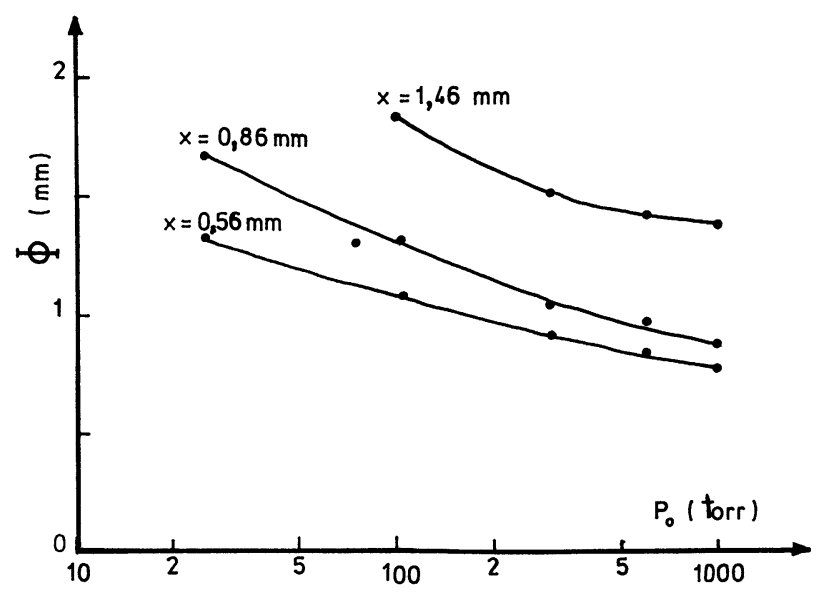

Fig. 3. - Largeur à mi-hauteur (ou diamètre $\Phi$ ) du jet de gaz en fonction de la pression d'admission $P_{0}$, à différentes distances $x$ de la buse.

[Full width at half maximum (or diameter $\Phi$ ) of the gas beam as a function of the pushing pressure $P_{0}$, for different distances $x$ from the nozzle.]

et $x$ sont représentées sur la figure 3 . On constate que, dans tout le domaine de pression d'admission considéré $(25$ à 1020 torr) le diamètre $\Phi$ à $x$ constant diminue au fur et à mesure que $P_{0}$ augmente, ce qui signifie que le jet est de mieux en mieux localisé vers son axe. Par ailleurs, la figure 4 donne la variation du diamètre $\Phi$ du jet en fonction de $x$, c'est-à-dire le long de l'axe du jet. Cette figure illustre bien à nouveau la directivité du jet obtenu, d'autant meilleure que $P_{0}$ est plus fort (noter le changement de courbure de la courbe à plus faible pression) et qui reste bonne même à des distances assez grandes de la buse. Sur la même figure, nous avons porté les résultats de mesures similaires effectuées à l'aide d'une buse simple [1], de $0,1 \mathrm{~mm}$ de diamètre : l'amélioration (en directivité mais non en diamètre) due au multicanal est évidente. Il est à noter que pour la buse simple le diamètre du jet est pratiquement indépendant de la pression d'admission.

L'extrapolation des courbes de la figure 4 vers le diamètre de la buse à $x=0$ nous permet de définir, par les tangentes à l'origine, un demi-angle $\delta \theta / 2$ d'ouverture du jet. La mesure de ces angles nous donne alors $6,8^{\circ}<\delta \theta<100^{\circ}$ pour $P_{0}$ variant entre 1020 et 75 torr. Des angles d'ouverture définis de cette manière ont été comparés dans I à la relation de Giordmaine et Wang [6]

$$
\theta_{1 / 2}=6,54 \sqrt{\frac{2 N}{a \bar{c}} \rho}
$$

où $\rho$ est le rayon moléculaire équivalent de collision, $N$ le flux de molécules à travers le tube, $a$ le diamètre de la buse, et $\bar{c}$ la vitesse moléculaire moyenne, relation qui n'est valable que dans le cas d'un écoulement en régime moléculaire où le libre parcours moyen $\lambda$ est supérieur au diamètre de la buse. Ce n'est pas le

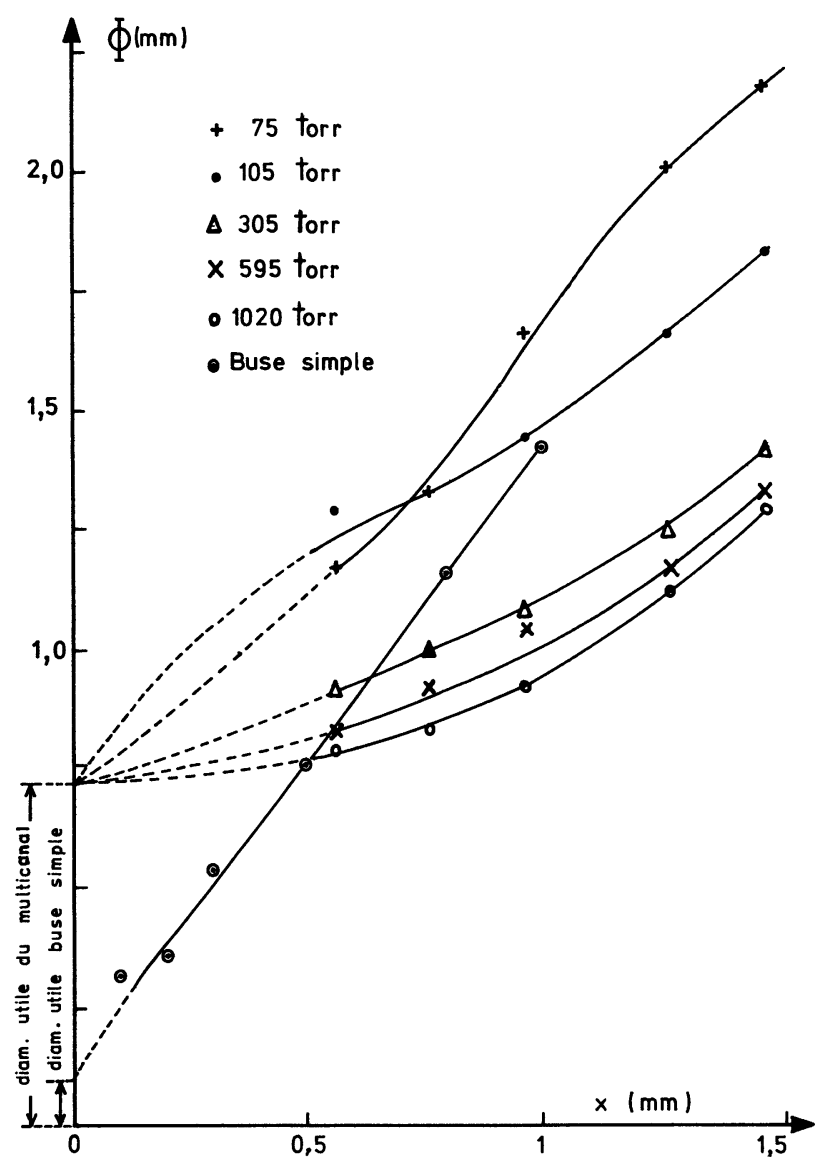

Fig. 4. - Largeur à mi-hauteur (ou diamètre $\Phi$ ) du jet de gaz en fonction de la distance $x$ entre buse et faisceau d'électrons, pour différentes pressions d'admission $P_{\mathrm{n}}$.

[Full width at half maximum (or diameter $\Phi$ ) of the gas beam as a function of the distance $x$ from nozzle to electron beam, for different pushing pressures $P_{0}$.]

cas ici, et nous verrons plus loin que dans nos conditions expérimentales l'écoulement est du type visqueux.

Afin de pouvoir établir une comparaison entre nos résultats expérimentaux et les seuls calculs existants concernant la directivité d'un jet multicanal dans le cas d'un écoulement visqueux [7], il est nécessaire d'introduire la distribution angulaire des molécules à la sortie de la buse en définissant la variable $\theta$ (Fig. 1b) par tg $\theta=y / x$. Cette distribution angulaire est alors donnée par les courbes expérimtentales $I / I_{0}=f(\theta)$ à pression d'admission $P_{0}$ constante (Fig. 5), dont la largeur à mi-hauteur peut constituer une nouvelle définition de l'angle d'ouverture du jet, désigné par $\Delta \theta$. On trouve alors que $\Delta \theta$ décroît de $87^{\circ}$ à $62,5^{\circ}$ quand $P_{0}$ augmente de 25 à 1020 torr. Il est à noter que ces distributions angulaires ne sont pas complètement indépendantes de $x, \Delta \theta$ diminuant légèrement, de quelques degrés, quand $x$ croît de 0,5 à $1,5 \mathrm{~mm}$. Ceci pourrait être dû à la difficulté de définir expérimentalement l'angle $\theta$ (voir discussion plus loin). Cependant cette variation de $\Delta \theta$ n'affecte pas sensiblement les conclusions exposées ci-dessous. 


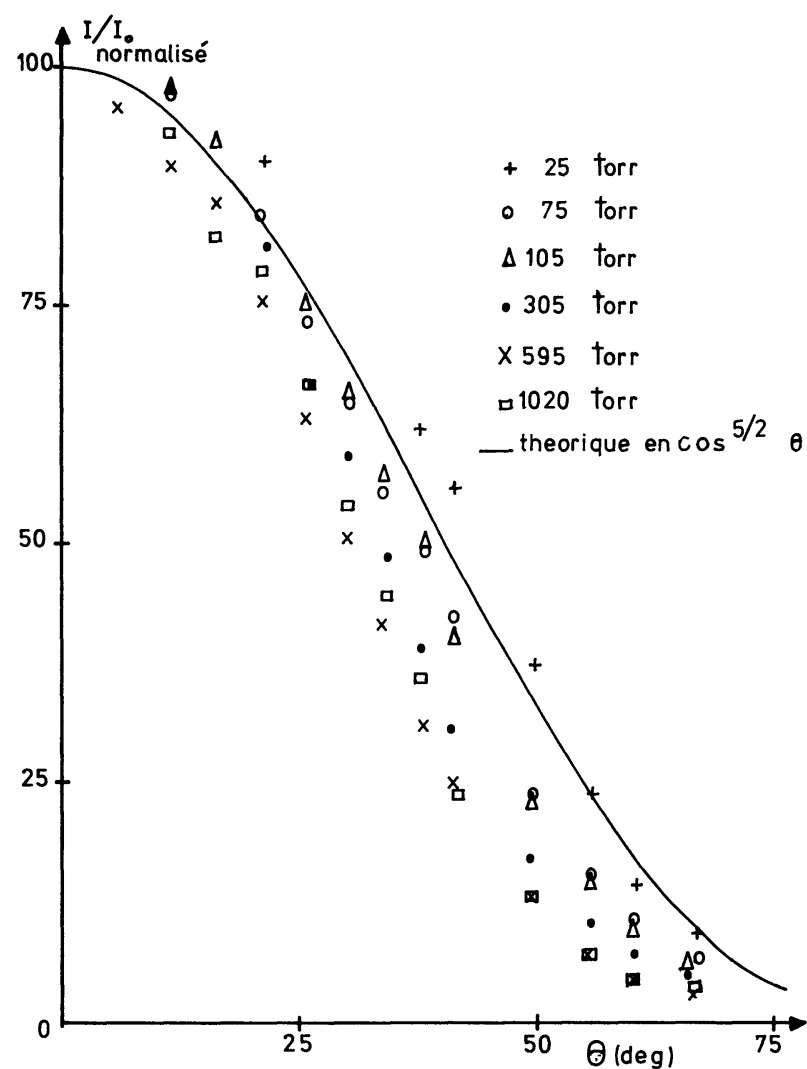

Fig. 5. - Distributions angulaires mesurées dans le jet, pour différentes pressions d'admission $P_{0}$ du gaz. La courbe en trait plein représente la prédiction théorique de Troitskii [7], valable pour les grandes valeurs de $P_{0}$.

[Angular distributions measured in the gas beam, for different pushing pressures $P_{0}$. The full line represents Troitskii's [7] calculations in the limit of large $P_{0}$ values.]

Dans son étude théorique, Troitskii [7] démontre que, en général, la largeur $\Delta \theta$ à mi-hauteur de la distribution angulaire dépend d'une manière non simple du rapport $a / L_{\text {eff }}$, où $a$ est le diamètre d'un canal de la buse et $L_{\text {eff }}$ est sa longueur efficace (1), c'est-à-dire que $\Delta \theta$ dépend de $P_{0}$ puisque $L_{\text {eff }}$ est lui-même une fonction de $P_{0}$ :

$$
L_{\text {eff }}^{3}=\left(2 \lambda_{0}\right)^{2} L=\frac{L}{2^{3} \rho^{4}}\left(\frac{k T}{\pi P_{0}}\right)^{2} .
$$

Toutefois Troitskii indique que dans le cas où $P_{0}$ devient suffisamment grand pour que $a / L_{\text {eff }} \gg 1$, la distribution angulaire tend vers une distribution limite, indépendante de $P_{0}$, et donnée par une loi en $\cos ^{5 / 2} \theta$ :

$$
I(\theta)=I(0) \cos ^{5 / 2} \theta
$$

dont la largeur à mi-hauteur, donnée par

$$
\cos ^{5 / 2} \frac{\Delta \theta}{2}=0,5
$$

est $\Delta \theta=81,45^{\circ}$. La distribution limite de Troitskii est également représentée figure 5 , et conduit qualita-

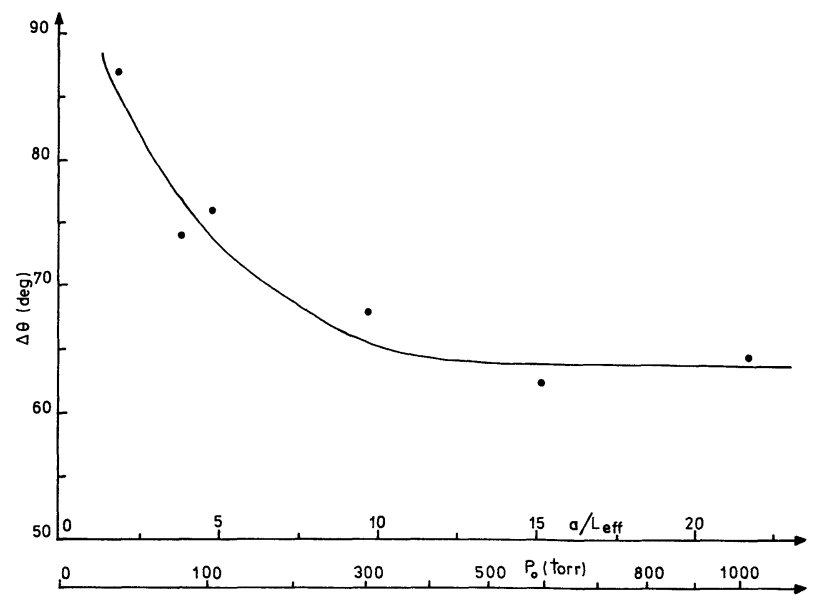

Fig. 6. - Variation de l'ouverture angulaire $\Delta \theta$ du jet de gaz, en fonction de la pression d'admission $P_{0}$ ou du paramètre $a / L_{\text {eff }}$ (voir texte)

[Variation of the angular divergence $\Delta \theta$ of the gas beam as a function of pushing pressure $P_{0}$ or parameter $a / L_{\text {eff }}$ (see text).]

tivement à un assez bon accord avec l'expérience. Ceci est mieux traduit par la figure 6 qui représente la variation de $\Delta \theta$ mesurée en fonction du paramètre $a / L_{\text {eff }}: \Delta \theta$ tend bien vers une limite quand $a / L_{\text {eff }}$ est $\gg 1$, mais cette limite n'est que de $\sim 63^{\circ}$. Plusieurs raisons peuvent être trouvées à ce désaccord quantitatif :

— raisons théoriques : Troitskii dans son calcul suppose pour simplifier que la pression à l'extrémité aval de la buse est nulle et néglige par ailleurs complètement les collisions intermoléculaires dans le jet après la sortie de la buse, même aux fortes pressions d'admission (ou grandes valeurs du paramètre $a / L_{\text {eff }}$ ) Son calcul étant établi pour une buse monocanal, cette hypothèse permet de l'étendre à une buse multicanal en considérant le jet ainsi formé comme une simple superposition de jets monocanal de même ouverture angulaire;

- raisons expérimentales : la définition de l'angle $\theta$ peut être entachée d'une certaine erreur (Fig. 1b) : d'une part du fait du diamètre non nul du faisceau d'électrons sonde, qui induit une incertitude sur l'origine de l'axe des $x$; d'autre part, alors que l'angle $\theta$ est défini dans le calcul théorique à partir du centre de la buse monocanal, nous avons arbitrairement défini $\theta$ ici à partir du centre de la buse multicanal, ce qui signifie que seules les molécules émises au voisinage de ce centre se trouvent en fait dans la direction $\theta$.

3.3 Densité DU JET. - Il a été précédemment montré $[1,8]$ que la densité volumique $n$ du jet le long de son axe (c'est-à-dire pour $y=0$ ), est reliée au rapport $I / I_{0}$ de l'intensité diffusée à l'intensité incidente (Fig. 1), par la relation

$$
I / I_{0}=n \sigma \Phi
$$

où $\sigma$ est l'intégrale, sur le domaine angulaire couvert 
par le capteur de diffusion, de la section efficace différentielle de diffusion des électrons par le gaz, c'est-à-dire

$$
\sigma=\int_{s_{\min }}^{s_{\max }} \frac{\lambda^{2}}{2 \pi} s \frac{\mathrm{d} \sigma}{\mathrm{d} \Omega} \mathrm{d} s .
$$

Dans le cas de $\mathrm{CO}_{2}, \sigma=8,0 \times 10^{-18} \mathrm{~cm}^{2}$ et dans le cas de $\mathrm{NH}_{3}, \sigma=1,65 \times 10^{-18} \mathrm{~cm}^{2}$.

A l'aide de ces relations, nous avons déterminé la densité $n$ dans le jet pour une distance buse-faisceau $x=0,56 \mathrm{~mm}$ : les résultats de nos mesures sont portés sur la figure 7 où l'on voit, en coordonnées logarithmiques, la variation de $n$ en fonction de la pression d'admission $P_{0}$, pour deux gaz différents, $\mathrm{CO}_{2}$ et $\mathrm{NH}_{3}$. Nous avons également gradué cette figure en pression équivalente dans le jet, déterminée en supposant ces gaz parfaits. La courbe de variation est, dans tout le domaine de pression considéré (25 à 4000 torr), une droite de pente 2 , ce qui signifie

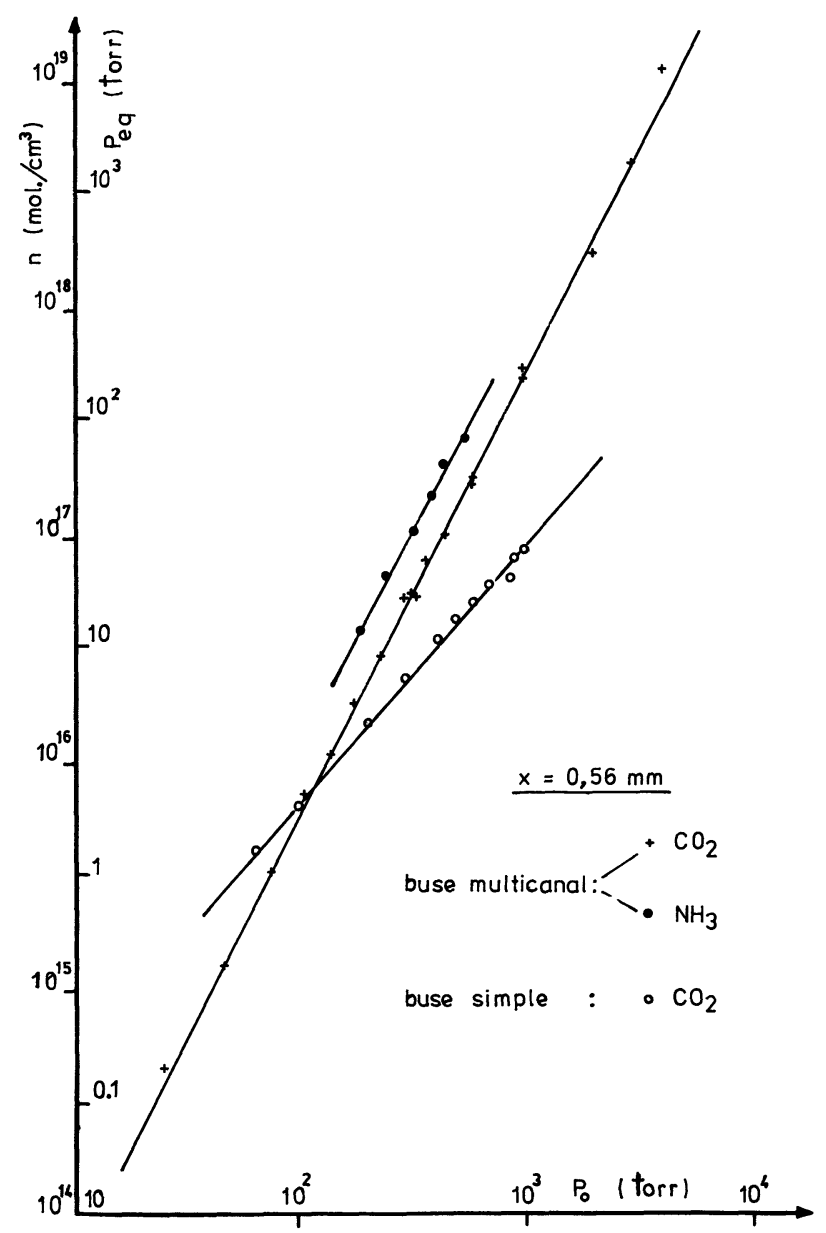

Fig. 7. - Densité moléculaire $n$ sur l'axe du jet formé par une buse multicanal et une buse simple, en fonction de la pression d'admission du gaz et pour une distance $x=0,56 \mathrm{~mm}$ entre buse et faisceau d'électrons. $P_{\text {eq }}$ représente la pression équivalente dans le jet.

[Molecular density, $n$, along the gas beam produced by a multichannel nozzle and a simple nozzle, versus pushing pressure $P_{0}$, at an electron beam to nozzle distance $x=0.56 \mathrm{~mm} . P_{\text {eq }}$ is the equivalent pressure in the gas beam.] que la densité moléculaire dans le jet est proportionnelle au carré de la pression d'admission. Sur la même figure sont représentés les résultats obtenus pour la buse simple décrite en I : la densité moléculaire est cette fois directement proportionnelle à $P_{0}$. Cette différence de comportement s'explique facilement si l'on admet, dans le domaine de pression d'admission considéré, des types d'écoulement différents dans les deux cas. En régime moléculaire [6,9], $n$ est en effet proportionnel à $P_{0}$. Par contre, Troitskii [7] a montré que, dans le cas d'un écoulement visqueux, si $P_{0}$ est suffisamment grand (ou $\left.a / L_{\text {eff }} \ll 1\right)$ pour que l'ouverture $\Delta \theta$ du jet ait atteint sa valeur limite (Fig. 6), alors la densité dans le jet est proportionnelle à $P_{0}^{2}$ :

$$
n=\mathrm{Cte} \times \frac{a^{4} P_{0}^{2}}{\eta L}
$$

où $\eta$ est le coefficient de viscosité du gaz considéré. Il semble donc bien que l'on ait affaire à un écoulement visqueux dans le cas du multicanal.

La formule précédente peut également être testée en regardant le rapport des densités obtenues avec $\mathrm{CO}_{2}$ et avec $\mathrm{NH}_{3}$, toutes conditions étant identiques. Ce rapport doit être tel que

$$
\frac{n_{\mathrm{CO}_{2}}}{n_{\mathrm{NH}_{3}}}=\frac{\eta_{\mathrm{NH}_{3}}}{\eta_{\mathrm{CO}_{2}}}=0,669
$$

avec

$$
\eta_{\mathrm{CO}_{2}}=14,5 \times 10^{-6} \mathrm{~N} . \mathrm{s} / \mathrm{m}^{2}
$$

et

$$
\eta_{\mathrm{NH}_{3}}=9,7 \times 10^{-6} \mathrm{~N} . \mathrm{s} / \mathrm{m}^{2}
$$

à $15^{\circ} \mathrm{C}$, d'après F. W. Sears [10]. Expérimentalement, nous trouvons un rapport égal à 0,56 , en accord relativement satisfaisant avec la valeur théorique, compte tenu des diverses approximations utilisées, notamment dans la détermination expérimentale du diamètre du jet ainsi que dans l'hypothèse de densité uniforme dans le jet.

Par ailleurs, la figure 7 montre également qu'à partir de $P_{0}$ supérieur à $\sim 200$ torr, la buse multicanal présente un gain notable en densité par rapport à la buse simple, allant jusqu'à un facteur environ 10 pour $P_{0}=1000$ torr.

Nous avons également mesuré la variation de la densité moléculaire le long de l'axe du jet. Les résultats de nos mesures sont portés sur la figure 8 où les courbes relevées à différentes pressions $P_{0}$ ont été arbitrairement normalisées entre elles à 100 pour $x=0,56 \mathrm{~mm}$. Une constatation s'impose à partir de ces courbes : sauf pour le cas $P_{0}=1020$ torr, la décroissance de la densité le long de l'axe est de moins en moins rapide au fur et à mesure que $P_{0}$ augmente. Ceci confirme bien qu'aux fortes pressions, le jet est mieux localisé vers son axe. 


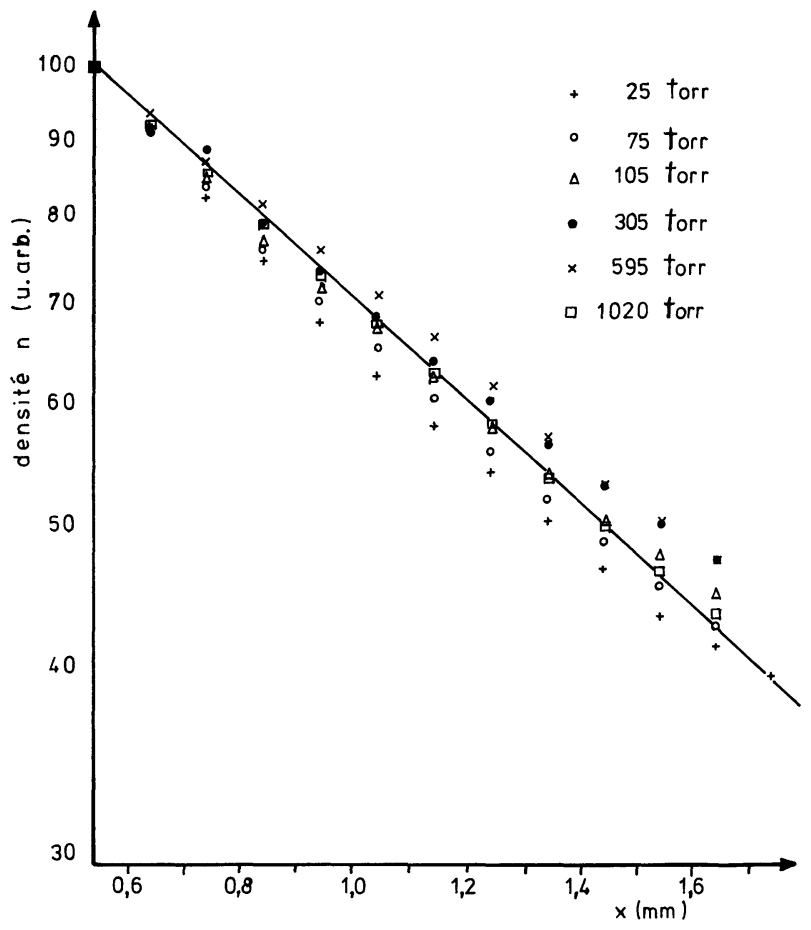

Fig. 8. - Densité moléculaire $n$ sur l'axe du jet formé par une buse multicanal, en fonction de la distance $x$ entre buse et faisceau d'électrons. En raison de l'accroissement quadratique de $n$ avec $P_{0}$ (Fig. 7), les courbes obtenues à différentes pressions d'admission $P_{0}$ ont été normalisées entre elles à $x=0,56 \mathrm{~mm}$, afin de permettre une comparaison de leur décroissance relative avec $x$.

[Molecular density, $n$, along the gas beam formed by a multichannel nozzle, versus electron beam to nozzle distance, $x$. Because of the quadratic increase of $n$ with $P_{0}$ (Fig. 7), the curves obtained at different pushing pressures $P_{0}$ have been renormalized to each other at $x=0.56 \mathrm{~mm}$ to allow comparison of their behaviour as a function of $x$.]

3.4 Formation D'AGRÉGATS DANS LE JET. - Les phénomènes de condensation dans les jets moléculaires ont déjà été mis en évidence par diffraction électronique, d'abord dans un générateur à haute intensité [11] où le faisceau moléculaire est extrait d'un jet supersonique au moyen d'un sélecteur, ensuite dans un jet supersonique libre issu d'une buse simple de $0,1 \mathrm{~mm}$ de diamètre [12]. Toutefois, alors que la plupart des auteurs (voir par ex. Farges [13]) cherchent à contrôler la formation de ces agrégats pour en étudier la structure interne, nous n'avons recherché pour notre part qu'à déterminer les conditions d'absence de ces agrégats dans le jet, afin de garantir pour nos expériences de collisions une cible qui reste monomoléculaire.

Nous avons pour cela répété l'expérience décrite en I, avec une plus grande finesse d'analyse [14], en étudiant en fonction de la pression d'admission $P_{0}$ la section efficace différentielle de diffusion élastique d'électrons de $35 \mathrm{keV}$ incidents sur le jet moléculaire $\left(\mathrm{CO}_{2}\right)$ issu de la buse multicanal. Cette répétition a été justifiée par les nombreuses améliorations apportées à la technique de mesure, et qui sont décrites en détail par ailleurs $[14,15]$. Les principales sont : i) une grande stabilité spatiale et en intensité du faisceau électronique incident, ii) l'asservissement de la rotation du canon à électrons qui balaie ainsi à vitesse constante le domaine angulaire choisi, ce qui permet d'obtenir directement toute la courbe de diffusion, à l'inverse des mesures point par point effectuées antérieurement, avec les fluctuations à court et long termes qu'elles entraînaient; iii) une précision largement accrue sur la connaissance de l'angle de diffusion, et iv) une meilleure résolution en énergie de l'analyseur électrostatique. La combinaison de toutes ces améliorations conduit à une réduction considérable des erreurs systématiques susceptibles d'avoir été présentes dans les mesures antérieures.

Les résultats de nos mesures sont portés sur la figure 9 pour des pressions d'admission variant de

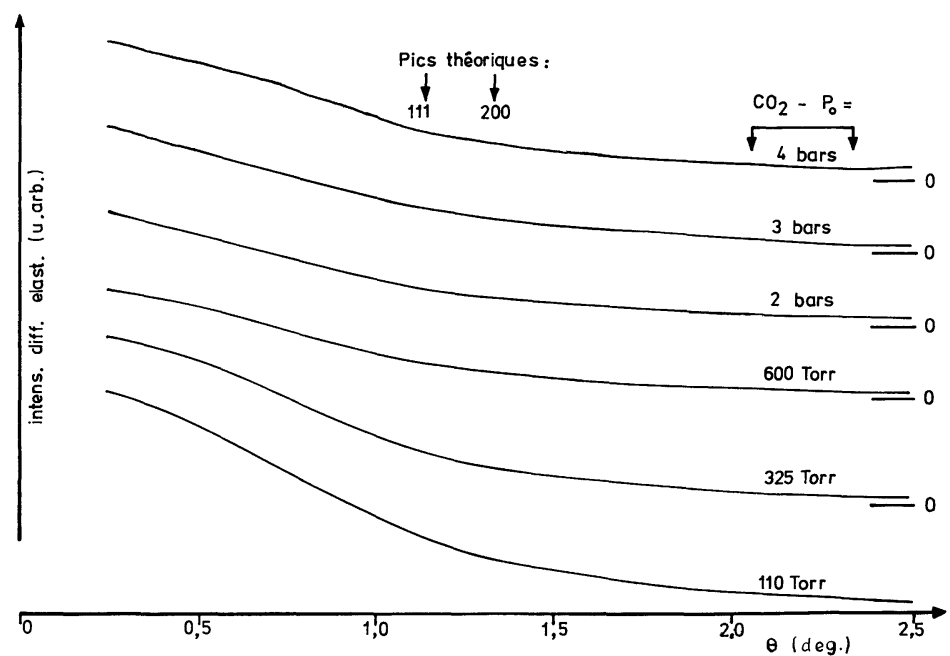

Fig. 9. - Section efficace différentielle de diffusion élastique d'électrons de $35 \mathrm{keV}$ par un jet de $\mathrm{CO}_{2}$ issu de la buse multicanal, en fonction de l'angle de diffusion $\theta$ et à différentes pressions d'admission $P_{0}$. Les flèches indiquent la position attendue pour les deux premiers pics cristallins de $\mathrm{CO}_{2}$.
[Differential cross section for $35 \mathrm{keV}$ electrons elastically scattered by a $\mathrm{CO}_{2}$ gas beam produced by the multichannel nozzle, versus scattering angle $\theta$, and for different pushing pressures $P_{0}$. The arrows indicate the expected positions of the first cristalline peaks of $\mathrm{CO}_{2}$.] 
100 torr à 4 bars. Il est évident d'après ces courbes qu'aucune structure se superposant à lintensité moléculaire et qui serait due à la diffusion par des cristaux de dioxyde de carbone n'est apparente. A titre d'indication, nous avons repéré sur cette figure les positions que l'on devrait attendre pour les deux premiers pics cristallins de $\mathrm{CO}_{2}$, déterminés par la relation de Bragg :

$$
\sin \left(\theta_{h k l} / 2\right)=\frac{\lambda}{2 a} \sqrt{h^{2}+k^{2}+l^{2}}
$$

où $\lambda$ est la longueur d'onde associée aux électrons, égale à $0,06447 \AA$ à $35 \mathrm{keV}$, et $a$ la maille du réseau cristallin, égale à $5,61 \AA$ pour $\mathrm{CO}_{2}$. Ces angles sont $\theta_{(111)}=1,14^{\circ}$ et $\theta_{(200)}=1,34^{\circ}$. On peut donc en conclure que, jusqu'à 4 bars, nous ne décelons pas d'agrégats dans notre jet et que celui-ci reste purement constitué de molécules libres. Cette conclusion annule les observations reportées en I, et qui peuvent avoir eu leur origine dans deux causes différentes : i) une trop grande proximité de la buse au faisceau d'électrons : au fur et à mesure que $P_{0}$ augmente, l'intensité électronique recueillie par la buse augmente également, donnant lieu à une diffusion par le métal de plus en plus importante. Dans ces nouvelles expériences, la buse est maintenue suffisamment loin du faisceau électronique pour que cette contribution reste négli- geable [15]; ii) un des isolants électriques du système Faraday-moniteur (Fig. 6 de I) était insuffisamment blindé et se chargeait très lentement sous l'effet des électrons diffusés, introduisant des perturbations locales dans la courbe de diffusion, d'autant plus marquées que la diffusion était plus importante, c'est-à-dire que $P_{0}$ était plus grand.

La comparaison des résultats obtenus ici avec ceux concernant la buse simple étudiée par Jaéglé [12] où la condensation se manifeste assez rapidement est de loin favorable à la buse multicanal quant à l'absence de condensation dans le jet.

En conclusion, nous pouvons résumer les caractéristiques de la buse multicanal comme suit :

- jet bien localisé autour de son axe avec un angle d'ouverture $\Delta \theta \sim 63^{\circ}$, pratiquement indépendant de $P_{0}$ à partir de $P_{0}$ supérieur à 200 à 300 torr ;

- très forte densité dans le jet, croissant quadratiquement avec $P_{0}$. Une densité de $10^{19}$ molécules $/ \mathrm{cm}^{3}$ a été obtenue à $P_{0}=4000$ torr à environ $0,5 \mathrm{~mm}$ de la buse, sans qu'ait pu être décelée aucune présence de microcristaux. (La distance $0,5 \mathrm{~mm}$ de la buse est notre position habituelle de travail où la diffusion par la buse elle-même est négligeable, $\lesssim 10^{-3}$.) Ceci est un avantage considérable dans les expériences de collisions où les sections efficaces sont faibles, l'augmentation de la densité de la cible ne devenant plus limitée que par le taux de diffusion multiple tolérable.

\section{Bibliographie}

[1] Nguyen, B., Lahmam-Bennani, A., Rouault, M., J. Phys. E8 (1975) 909.

[2] Livshits, A. I., Metter, I. M., Rikenglaz, L. E., Sov. Phys. Tech. Phys. 16 (1971) 276.

[3] Neudachin, I. G., Porodnov, B. T., Suetin, P. E., Sov. Phys. Tech. Phys. 17 (1972) 848.

[4] Yuferov, V. B., Skibenko, E. I., Kholod, Yu. V., Il'chenko, A. M., Bredikhin, M. Yu., Maslov, A. I., Sov. Phys. Tech. Phys. 16 (1972) 1341

[5] Ivanov, B. S., TroitskiI, V. S., Sov. Phys. Tech. Phys. 8 (1963) 365.

[6] Giordmaine, J. A., J. Appl. Phys. 31 (1960) 463.

[7] TroitskiI, V. S., Sov. Phys. Tech. Phys. 7 (1962) 353.

[8] Audit, P., Rouault, M., Entropie 18 (1967) 22.
[9] Bonham, R. A., Fink, M., High Energy Electron Scattering (Van Nostrand, N.Y.) 1974

[10] Sears, F. W., Thermodynamics (Addison Wesley Pub. Comp.), 2nd ed., 1968.

[11] Audit, P., Rouault, M., C.R. Hebd. Séan. Acad. Sci. 265B (1967) 1100

[12] Jaegle, A., Duguet, A., Rouault, M., C.R. Hebd. Séan. Acad. Sci. 267B (1968) 1081.

[13] Farges, J., Raoult, B., Torchet, G., J. .Chem. Phys. 59 (1973) 3454

[14] Lahmam-Bennani, A., Duguet, A., Wellenstein, H. F., J. Phys. B, accepté pour publication (1978).

[15] Lahmam-Bennani, A., Thèse de Doctorat, Université de Paris-Sud, Orsay, 1978. 